\title{
Internacionalização da educação superior: tensões, cenários e estratégias nos casos Brasil e Espanha
}

\author{
Maria da Salete de Farias, João de Oliveira \\ \& Luis Lorente
}

\begin{abstract}
Resumo
O presente artigo apresenta reflexões decorrentes da realização de estágio pós doutoral que objetivou conhecer experiências sobre o processo de internacionalização da educação superior no Brasil e Espanha, considerando objetivos, estratégias e tensões. Para tanto, foi realizada revisão bibliográfica e analisados documentos relevantes sobre o tema, a exemplo da proposta da Coordenação de Aperfeiçoamento de Pessoal de Nível Superior (Capes) através do Edital n 41/2017, disponível na WEB Capes (2017) e o documento Estrategia para La Internacionalización de las Universidades Españolas 2015-2020. A teoria de campo científico de Bourdieu nos possibilitou ver que as estratégias utilizadas para a internacionalização da educação superior, mais acentuadas nos tempos atuais, se inserem também no jogo de lutas pelo poder e pelo monopólio da autoridade científica entre instituições acadêmicas nacionais e internacionais.
\end{abstract}

Palavras-chave:

internacionalização; educação superior; campo científico. 


\section{Internacionalización de la educación superior: tensiones, escenarios y estrategias en los casos Brasil y España}

Resumen: Este artículo presenta reflexiones derivadas de estudios post doctoral que tuvo como objetivo conocer experiencias sobre el proceso de internacionalización de la educación superior en Brasil y España, considerando objetivos, estrategias y tensiones. Con este fin, se realizó una revisión de la literatura y se analizaron documentos relevantes sobre el tema, como la propuesta de la Coordinación para la Mejora del Personal de Educación Superior (Capes) a través del Aviso Público No. 41/2017, disponible en WEB Capes (2017) y el documento Estrategia para la internacionalización de las universidades españolas 2015-2020. La teoría del campo científico de Bourdieu nos ha permitido ver que las estrategias utilizadas para la internacionalización de la educación superior, más destacadas en los tiempos actuales, también son parte de la lucha de poder y el monopolio de la autoridad científica entre las instituciones académicas nacionales e internacionales.

Palabras clave: internacionalización; educación universitaria; campo científico.

\section{Internationalization of higher education: voltages, scenarios and strategies in Brazil and Spain cases}

Abstract: This article presents reflections derived from the post-doctoral internship that aimed to learn about the process of internationalization of higher education in Brazil and Spain, considering objectives, strategies and tensions. For this purpose, a literature review was conducted and relevant documents on the subject were analyzed, such as the proposal of the Coordination for the Improvement of Higher Education Personnel (Capes) through Public Notice No. 41/2017, available at WEB Capes (2017) and the document Strategy for the internationalization of Spanish universities 2015-2020. Bourdieu's theory in the scientific field has allowed us to see that the strategies used for the internationalization of higher education, most outstanding modern times, are also part of the power struggle and the monopoly of scientific authority among national and international academic institutions.

Keywords: internationalization; university education; scientific field.

\section{L'internationalisation de l'enseignement supérieur: tensions, scénarios et stratégies dans les affaires Brésil et Espagne}

Résumé : Cet article présente les réflexions découlant du stage postdoctoral qui visait à connaître les expériences relatives au processus d'internationalisation de l'enseignement supérieur au Brésil et en Espagne, en tenant compte des objectifs, des stratégies et des tensions. À cette fin, une revue de la littérature a été réalisée et des documents pertinents sur le sujet ont été analysés, tels que la proposition de la Coordination pour l'amélioration du personnel de l'enseignement supérieur (Capes) par l'Avis au public $n{ }^{\circ} 41 / 2017$, disponible sur WEB Capes (2017) et le document Stratégie pour l'internationalisation des universités espagnoles 2015-2020. La théorie scientifique de Bourdieu sur le terrain nous a permis de voir que les stratégies utilisées pour l'internationalisation de l'enseignement supérieur, plus accentuées de nos jours, s'inscrivent également dans les luttes de pouvoir et le monopole de l'autorité scientifique entre les établissements universitaires nationaux et internationaux.

Mots-clés: internationalisation; enseignement supérieur; domaine scientifique. 


\section{Introdução}

O tema da internacionalização da educação superior, em que pese volume considerável de publicações enfocando o assunto, ainda requer estudos sobre seu sentido, sua dinâmica e impacto nas instituições de ensino superior (IES) e, sobretudo, na gestão universitária e no trabalho acadêmico. Ele se encontra articulado com os processos de globalização, com a agenda e momento histórico educativo, em geral, e com as trajetórias das reformas e políticas educacionais em cada país.

Relacionando a internacionalização da educação com a globalização, Santos (2002: 52) mostra o duplo efeito que ela provoca, se por um lado, os processos de transformações globalizados vêm aumentando interações transnacionais, por outro, também tem gerado desigualdades em grande escala tanto locais quanto globais, tanto entre pessoas como entre países. Romão (2008: 169), por sua vez, confirma o pensamento de Santos (2002) ao dizer que a globalização trouxe complexas relações entre os países, criando novas conexões, intensificando desigualdades com desdobramentos também nos sistemas educacionais. Neste contexto da globalização, a educação torna-se imperativa para o entendimento de novas perspectivas resultantes do entrelaçamento com diferentes culturas para sua compreensão, convivência e alargamento de teorias e práticas mediante, principalmente, a produção de novos conhecimentos.

Para Bianchetti e Sguissardi (2017), a universidade, devido as características históricas que Ihe são inerentes, passa a ser considerada na sociedade capitalista como um instrumento subordinado à lógica econômica; a educação superior, e em particular a universidade, passa a ser percebida cada vez mais a partir do grande potencial para se transformar em um dos mais vibrantes mercados no século XXI, tendo em vista poder contribuir com o desenvolvimento econômico das nações por meio da internacionalização acadêmica e financeira. A educação superior, tanto na graduação como na pós-graduação, vem se tornando um setor de serviços, globalizando-se e mercadorizando-se rapidamente por meio da oferta de cursos e produtos acadêmicos. Para Marim, (2004: 13), "a internacionalização da educação superior [...] vem ampliando o interesse por seu entendimento em razão das consequências quanto à formação dos grandes blocos econômicos que buscam flexibilizar o comércio e o consumo entre os continentes".

No que tange às ações de intercâmbio e cooperação entre pares nacionais e internacionais, estes se configuram como processos repletos de intencionalidades ou racionalidades, como nos chama a atenção Azevedo e Catani (2015: 71), ao dizer que "[...] a análise do fenômeno da internacionalização da educação superior [...] à luz da contradição, pode adquirir o sentido humanista da solidariedade e da civilidade ou o sentido coisificante da expansão capitalista e da mercadorização" [...]. Com base em 
Bourdieu (2006), podemos dizer tratar-se de um espaço ou campo social onde interações, transições e eventos ocorrem de modo cada vez mais intenso.

Vemos a partir da compreensão de Bourdieu (1983) que o campo científico é um espaço de luta pelo monopólio da autoridade científica em escala nacional ou internacional, o que nos leva a inferir que as estratégias de internacionalização adotadas no contexto atual instigam diferentes agentes a proporem ações que visam o monopólio do conhecimento, com desdobramentos epistemológicos, sociais e políticos. Afinal, para Bourdieu (2011) todo campo constitui sempre um espaço de forças e de lutas com interesses específicos. Como espaço de disputa, os agentes usam de diferentes estratégias para manter ou alterar seu capital e sua posição do campo, seja ele o campo científico, campo econômico ou campo do poder. Nessa direção, a internacionalização vem se tornando um critério de distinção dos agentes que atuam no campo científico ou universitário, o que se observa por meio de cursos, programas, universidades, produção científica ou mesmo habitus dos pesquisadores.

É com esta percepção que este artigo aborda o tema da internacionalização, considerando os contextos brasileiro e espanhol e suas recentes políticas e estratégias. Num primeiro momento apresentamos estratos do processo de internacionalização no caso brasileiro e, num segundo momento, tecemos considerações acerca do caso espanhol. Ao final, recorrendo à teoria de campo científico de Bourdieu (1983) buscamos mostrar que as políticas de internacionalização, tanto no Brasil como na Espanha, estão eivadas de racionalidades cujas estratégias e ações procuram firmar a legitimidade acadêmica, o poder e a competição entre profissionais, instituições, grupos, nações e blocos econômicos.

\section{Internacionalização da Educação Superior no Brasil: a Pós-Graduação em perspectiva}

O cenário do mundo globalizado, ao considerar a interdependência entre os países e os desafios na perspectiva de promover intercâmbios e acelerar a cooperação internacional levou principalmente as universidades no Brasil a gradativamente fortalecerem seus investimentos em políticas de internacionalização. Isto vem se dando mediante a efetivação de processos de cooperação internacional em suas diferentes feituras, como: Programas de Mobilidade de pesquisadores, professores e estudantes, Redes de Cooperação Internacional, Acordos de Colaboração, visando à organização de projetos internacionais na área da ciência e tecnologia e o fortalecimento das relações entre as IES de diferentes países. Para estes fins, a pós-graduação tem sido uma mola propulsora dessas estratégias e ações, como se pode evidenciar nas propostas dos planos Nacionais de Pós-Graduação no Brasil. 
É sabido que as discussões sobre a internacionalização da educação superior foram intensificadas a partir de orientações das Conferências Mundial sobre Educação Superior ocorridas nas décadas de 1990 e 2000, convocadas pela Organização das Nações Unidas para a Educação, a Ciência, e a Cultura (Unesco). Estas conferencias foram também marcadas pela presença constante de organismos multilaterais, a exemplo do Banco Mundial (BM) e da Organização Mundial do Comércio (OMC), no papel de indutores de estabelecimentos de acordos entre países e continentes na perspectiva do compartilhamento de conhecimentos teóricos e práticos.

A pós-graduação brasileira, sobretudo nas universidades públicas, foi gradativamente se fortalecendo, desde da segunda metade dos anos 1960, mediante ações de duas agências governamentais de fomento e avaliação: a Coordenação de Aperfeiçoamento de Pessoal de Nível Superior (Capes) e o Conselho Nacional de Desenvolvimento Científico e Tecnológico (CNPq). Ambas agências foram fundamentais no processo de institucionalização e consolidação da pesquisa e da pós-graduação no país. Atualmente, de acordo com Marrara (2007: 251) a pós-graduação vem se apresentando "como um mecanismo essencial, não somente para a formação acadêmica (discente e docente), como também para a solução de problemas brasileiros e comuns da humanidade".

Nessa direção, o Plano Nacional de Pós-Graduação - PNPG (2005-2010), no seu item 4.5, afirmava que a cooperação internacional por intermédio das universidades, deverá ser estimulada de tal forma que o intercâmbio entre alunos e professores fosse institucionalizado, incentivando-se inclusive a apresentação de projetos de captação de recursos junto às agências de fomento. Este Plano deve ser inserido em estratégias de desenvolvimento econômico e social do país (Brasil, 2004: 61; 89), a exemplos dos anteriores. A institucionalização, expansão e consolidação da pós-graduação brasileira, além das ações da Capes e do CNPq, deve muito de seu sucesso aos Planos que garantiram a implementação de uma política de Estado para o setor, sendo o primeiro PNPG datado do período 1975-1979.

Por sua vez, o Plano Nacional de Educação- PNE (2014-2024) reafirma o incentivo às relações internacionais da educação superior, e institui, na Meta 14, assim como na meta 13, estratégias para consolidar cursos, programas, projetos e ações que objetivem a formação e titulação pós-graduada e a internacionalização da pesquisa e da produção do conhecimento; estimula ainda a atuação em rede e o fortalecimento de grupos de pesquisa (estratégia 14.6); promoção do intercâmbio científico e tecnológico, nacional e internacional, entre as instituições de ensino, pesquisa e extensão (estratégia 14.9).

O PNPG 2011-2020 também enfatiza o combate às assimetrias regionais, ênfase na inclusão social e "estímulo à formação de redes de pesquisa e pós-graduação, envolvendo parecerias, nacionais e internacionais, no nível da fronteira do conhecimento, com 
vistas à descoberta do 'novo' e do inédito". Com essa diretriz espera-se, mediante a internacionalização da pós-graduação, ampliar a excelência do ensino e das pesquisas no país. Esse mesmo PNPG traz com clareza elementos para se pensar e dar sequência ao processo de internacionalização da educação superior, em nível de pós-graduação. O item 2.3.5, por exemplo, orienta políticas de cooperação internacional e de formação de recursos humanos no exterior recomendando "estimular a cooperação internacional por intermédio das universidades, de tal forma que o intercâmbio entre alunos e professores fosse institucionalizado permitindo inclusive a apresentação de projetos de captação de recursos junto às agências de fomento internacionais (Brasil, 2010: 35).

As modalidades de cooperação internacional como ampliação do atual modelo e as parcerias institucionais, envolveriam intercâmbios recíprocos de alunos e professores em projetos de pesquisa específicos, bolsas-sanduíche para os alunos, estágios de curto prazo para professores e estágios para recém doutores. Prevê também a intensificação dos programas de intercâmbio, visando ao compartilhamento na orientação de doutorandos com pesquisadores atuando no exterior em áreas de interesse estratégico para o país, além do apoio a estágio de pós-doutoramento para jovens doutores, tendo por base a qualidade do projeto a ser desenvolvido. Foca na ampliação do intercâmbio institucional de estudantes de graduação, visando a seu futuro ingresso na pós-graduação; o estímulo a parcerias e formação de redes de pesquisa na cooperação Sul-Sul, como suporte à formação de recursos humanos em áreas prioritárias e de interesse comum (Brasil, 2010: 37).

O PNPG (2011-2020) dedica ainda um capítulo especial a Internacionalização da Pós-Graduação e a Cooperação Internacional (Presença Internacional da Ciência e da Tecnologia Brasileira), mostrando resultados comparativos com a produção científica mundial, sinalizando para a presença da ciência brasileira no cenário internacional. Conforme o Plano pode ser aferida, senão quantitativamente, mas pelo menos qualitativamente, pela atuação de cientistas brasileiros nas principais instituições internacionais de ciência, frequentemente em posições de destaque, através de notáveis ações de cooperação científica internacional promovidas pelas agências de fomento, federais e estaduais (Brasil, 2010: 231).

Em 2011, a internacionalização da educação brasileira, passa a configurar de forma mais intensa e destacada pelas políticas implantadas pelo governo federal. É nesse período que ela se oficializa mais fortemente como uma estratégia de incentivo às IES e centros de pesquisa no país (Brasil, 2011), ao proporcionar aos estudantes e professores oportunidades para realizarem estudos em universidades estrangeiras com tradição em pesquisa e grande capacidade de inovação, favorecendo a cooperação científica internacional. Merece registro o Programa Ciência sem Fronteiras, instituído pelo Decreto $n^{\circ}$ 7.642, de 13 de dezembro de 2011, iniciativa do Governo Federal por meio dos Ministérios da Ciência, Tecnologia e Inovação (MCTI) e Ministério da 
Educação (MEC) e suas instituições de fomento - CNPq e Capes. Dado a crise econômica e as mudanças ocorridas no país, o Programa foi encerrado em 2017.

Em fevereiro de 2017, durante o governo de Michel Temer, a Capes lançou o "Programa Mais Ciência Mais Desenvolvimento para as IES". Para tanto, a Capes enviou, sobretudo para as universidades federais, questionário diagnóstico e prospectivo sobre a capacidade de internacionalização das instituições, com o compromisso de elas apresentarem um programa próprio de internacionalização, tendo como eixo principal a pós-graduação stricto sensu. Cada plano deveria contemplar diferentes etapas, objetivos, estratégias e metas de ação, uma 'espécie' de "Plano Institucional de Internacionalização". Na sequência, a Capes lançou o Programa de Internacionalização Capes-Prlnt mediante Edital n 41/2017 visando "fomentar o desenvolvimento de Planos Estratégicos de Internacionalização como meio de melhorar a qualidade dos cursos de pós-graduação brasileiros e de conferir maior visibilidade à pesquisa científica realizada no Brasil”. Na primeira fase do PrInt (análise documental) foram aprovados 108 IES/Institutos de Pesquisas. Na fase final (análise de mérito) foram aprovados apenas 36. A aprovação veio acompanhada em cada IES de propostas de incentivos para incrementar a produção acadêmica e científica dos seus programas de pós-graduação.

\section{A Internacionalização da Educação Superior no Espaço Europeu: ecos nas Universidades Espanholas}

No âmbito da internacionalização da educação superior as universidades espanholas recebem também influências de organismos multilaterais (Unesco, Banco Mundial, OCDE) que estabelecem a chamada Agenda Educativa Mundial (AEM) desmembrados em diversos programas.

Programas como PISA (Programmefor International StudentAssessment) de la OCDE, TIMSS (Trends in International Mathematics and ScienceStudy), o PIRLS (Progress in International Reading LiteracyStudy) del IEA International Study Center, son un excelente ejemplo de la penetración del enfoque comparado en educación desde una perspectiva global ofreciendo a los académicos e investigadores de nuestro campo una excelente herramienta susceptible de uso tanto en la docencia como en la investigación (Lazaro, 2018: 3).

No lastro destes programas ganham força organismos supranacionais que, no caso espanhol, têm sua visibilidade nas orientações da União Europeia, com vistas a incrementar as condições de produção e comercialização, bem como reduzir as desigualdades sociais econômicas entre o local e o regional. A União Europeia (UE) considerada um organismo supranacional também recorre às IES para fomentar políticas, 
ações estratégias na direção do crescimento econômico no atual contexto da globalização. Ela é vista como um importante instrumento para promover o desenvolvimento da qualidade das universidades espanholas "teniendo el potencial de ser el motor para la reforma del sistema universitário español en busca de La mejora de sueficiencia, excelencia y competitividade nun entorno global” (UE, 2016: 6).

A orientação oferecida por esta instituição é a de que se faz necessário ter um sistema de educação superior internacionalizado de forma a propiciar a melhoria qualitativa de professores altamente qualificados de modo que estes em sua prática profissional possam atender as demandas que a economia global e sociedade requerem. O ensino deverá fornecer aos estudantes qualidade, competências e habilidades para atuarem no mercado competitivo e para isso o sistema educativo deverá oferecer "educación internacional de calidad mundial em inglés y otras lenguas extranjeras" (UE, 2016: 6). Uma das exigências do sistema educacional europeu tem sido tornar as universidades espanholas cada vez mais atraentes e reconhecidas internacionalmente para poder competir "com as melhores universidades de outros países [...].

O Processo de Bolonha tem jogado papel fundamental no crescente processo de internacionalização do ensino superior europeu que se encontra em marcha desde 1999, completando agora vinte anos (EU, 2018). Desde o início, os principais objetivos do Processo de Bolonha, especificamente os aspectos de harmonização e mobilidade, têm ressaltado o interesse em internacionalizar os sistemas nacionais de educação superior na Europa (Craciun, 2018), entretanto de acordo com a análise de Zgaga, Teichler, Brennan (2013) o processo vem acontecendo num equilíbrio nem sempre fácil entre centro e periferia. Dentre as medidas tomadas, em nível de espaço europeu, destacamos o acordo celebrado em 2007 pelos ministros dos 47 países incluídos no processo de Bolonha, denominado "una Estrategia para La Dimensión Exterior del EEES y en 2012 una Estrategia de Movilidad". Em 2013, a UE aprovou uma estratégia de internacionalização para dar concretude à mobilidade internacional de estudantes e pessoas nos sistemas de educação superior.

Tomando por base análise do documento da Estrategia para La Internacionalización de las Universidades Españolas 2015-2020, publicado em março de 2015, pelo Ministério de Educação, Cultura e Desporto, nota-se que este apresenta como objetivo,

Consolidar un sistema universitario fuerte e internacionalmente atractivo que promueva la movilidad de entrada y salida de los mejores estudiantes, profesores, investigadores y personal de administración y servicios, la calidad educativa, el potencial del español como idioma para la educación superior, la internacionalización de los programas formativos y las actividades de I+D+i, contribuyendo a la mejora del atractivo y de la competitividad internacional de España, así como al desarrollo socioeconómico de su entorno próximo basado en el conocimiento (2015: 7) 
Foram estabelecidos quatro eixos estratégicos: a) Consolidar un sistema universitario altamente internacionalizado. b) Aumentar el atractivo internacional de las universidades. c) Promover la competitividad internacional del entorno. d) Intensificar la cooperación en educación superior con otras regiones del mundo (UE, 2016: 8-10).

O primeiro eixo busca reforçar a ideia de formar competências e habilidades necessárias ao trabalho internacional e aberto, mediante a formação de docentes e investigadores com experiência internacional para favorecer a internacionalização universitária. Neste sentido, o documento convida à melhoria do marco legal e das condições para a internacionalização das universidades em nível estatal e regional. Outra ação deste eixo refere-se à internacionalização dos programas formativos e à mobilidade nos programas de estudos de grado y máster. Destaque-se também a recomendação da 'Internacionalización en casa1 - que tem por objetivo incrementar o "multilinguismo, la multiculturalidad y la internacionalización de los campus universitarios españoles". O eixo ainda aponta para a melhoria da eficiência e eficácia no "reconocimiento de títulos y períodos de estúdio' para estudantes do Espaço Europeu de Ensino Superior em conformidade com a Convenção de Lisboa sobre o Reconhecimento (UE, 2016: 9.8). Para tanto tem sido adotado instrumentos a nível europeu, nacional, regional e institucional com o objetivo de facilitar o reconhecimento equitativo das qualificações estrangeiras e/ou períodos de estudo no estrangeiro (Rede Eurydice, 2012: 55).

O segundo eixo trata do aumento do 'atractivo internacional de las universidades' considerando como atividade atrativa a acolhida de estudantes estrangeiros e oferta de formação em inglês e outras línguas estrangeiras além do fomento à participação dos estudantes e professores em redes, projetos e programas internacionais.

Já o terceiro eixo se volta para a promoção 'de La competitividad internacional del entorno' devendo ser operacionalizado mediante a participação nas redes internacionais de conhecimento com o propósito de dinamizar o progresso e intercambiar saberes, ciência, tecnologia e inovação. É importante também a aproximação com o meio industrial e empresarial "para fomentar La participación conjunta em proyectos y programas internacionales de prácticas de estudiantes, doctorados industriales, liderazgo industrial y innovación"(UE, 2016: 9.10)

O quarto eixo trata 'de La intensificación de La cooperación em educación superior com otras regiones del mundo', incentiva as possibilidades de integração nos programas de grado e master com o estabelecimento de colaboração e acordos bilaterais envolvendo o espaço Iberoamericano de Conhecimento, o Espaço Euro mediterrâneo de Educação Superior e Investigação com países desenvolvidos e emergentes. A globalização intensifica a necessidade de conhecimento para o desenvolvimento dos países em níveis local, regional e mundial.

Dentre outras ações destaca-se o apoio e a credibilidade dada ao Program European Action Scheme for the Mobilityof University Students (Erasmus) que viabiliza a 
execução de projectos de cooperação transnacional dando assim maior visibilidade a agenda política da UE no tocante a internacionalização da Educação Superior.

Como se vê, a estratégia de internacionalização das Universidades Espanholas ao seguirem as orientações da UE, seus objetivos e metas, centra no desenvolvimento de medidas de mobilidade internacional de estudantes de graduação e pós graduação mediatizados principalmente pelo - Erasmus. Neste sentido é emblemático o recente anuncio da Comissão Européia em 26 de junho de 2019 de que das 54 solicitações recebidas pelo Erasmus, foram selecionadas 17 instituições de ensino superior de toda a Europa para formarem parte das primeiras alianças de 'Universidades Européias' que se constituirão em modelos para todas as outras da União Européia. As Universidades Européias selecionadas passarão a ser campus universitários onde estudantes e professores terão a oportunidade de viver experiências de Europa estudando em distintos países colocando em comum conhecimentos especializados, plataformas e recursos [...].A iniciativa pretende 'reforzar significativamente La movilidad de los estudiantes y el personal, así como fomentar La calidad, La inclusión y La competitividad de La enseñanza superior europea'. Assim está em curso a Rede de Universidades Européias, com o objetivo de reunir uma nova geração de europeus criativos e capazes de cooperar "más allá de lãs lenguas, lãs fronteras y lãs disciplinas", para fazer frente aos grandes desafios sociais e a escassez de capacidades enfrentadas pela Europa.

Esse Programa visa que os estudantes graduem-se combinando períodos de estudos em vários países da União Européia de forma a contribuir com a competitividade internacional do conjunto das Universidades desta Comunidade. Esse leque de ações e estratégias dimensionadas pela geografia do conhecimento científico, pelo estímulo a competitividade, via Rede de Universidades Europeias, faz parte do jogo da luta científica ao impor a comunidade científica normas e valores para que se sobressaiam e enfrentem os desafios com criatividade, qualidade e competitividade. Na era do conhecimento, próprio dos processos contemporâneos de globalização, a racionalidade traz demandas aos estados e aos blocos econômicos para a produção e investimento que inspirem concorrências ou vantagens competitivas, não sendo à toa e comum que se recorra à educação para dá conta das exigências da sociedade cada vez mais alicerçada na competição, habilidades, competências, flexibilidade, criatividade e inovação. Neste ponto, como diz Ruano-Borbalan (2011: 444), "estabelecem-se os marcos legislativos, institucionais e curriculares, além de organizar o conjunto dos sistemas de apoio, parcerias e abertura a atores privados".

A monopolização do conhecimento se ramificará em todas as situações políticas, sociais e culturais tornando-se um referencial distintivo entre outras comunidades geopolíticas do mundo. Ou seja, as estratégias de internacionalização estão contidas de valores, visões de mundo, posições políticas e relações de poder a serem reproduzidas 
e legitimadas. Reforça essa ideia da não neutralidade do campo científico, as palavras de Azevedo (2017: 298) quando ele argumenta que,

A educação superior pressupõe, para além do pensamento nacional e as instituições que lhe dão corpo, a circulação internacional das ideias que, por sua vez, frise-se, não se trata de um fenômeno social neutro, carrega consigo contradições (público $\times$ privado, nacional $\times$ internacional solidariedade internacional $\times$ imperialismo cultural etc.).

De modo geral, vemos que a educação internacionalizada pode ser considerada assim um espaço ou campo social de disputa hegemônica onde desenvolve ações e estratégias situadas no campo universitário, de modo articulado ao campo econômico e ao campo do poder, formando campos sociais múltiplos e estabelecendo relações de força capazes de regularem práticas e processos que geram dependências tanto locais como mundiais. O tipo de escolaridade ou de formação recebida pelas pessoas na conjuntura atual educacional como suporte econômico de competências, habilidades e competitividade, influencia diferentes campos sociais na perspectiva da economia, da cultura e das sociabilidades.

Nesse contexto, vale lembrar que para Bourdieu (2011: 197) cada campo social possui intersecções com outros campos sociais e integra um espaço ou mundo social mais amplo, cujas ações e estratégias ao se manifestarem atendem a interesses específicos, locais, nacionais e até mundiais, com o agravamento de que são impostas e ao mesmo tempo consentidas em sua operacionalização por diferentes agentes sejam pessoas ou instituições. A análise de Bourdieu sobre os modos que o sistema educacional opera para classificar, selecionar, eleger e tornar distinto universidades e IES, em geral, para dar concretude a internacionalização, implica na construção de processos e dinâmicas de luta pela apreensão do poder mediante corrida pelo controle do conhecimento. Entendemos que a luta, resultado de processos, cooperação e colaboração, deveria ser sim, para a qualificação humana, para o avanço do conhecimento e resolução de grandes problemas sociais, ambientais e outros e não para atender basicamente a fins mercadológicos, regidos pela funcionabilidade inerente à lógica do capital cada vez mais globalizado.

\section{Considerações Finais}

A análise empreendida procurou mostrar como a intensificação do debate e da busca pela internacionalização da educação superior tanto no Brasil como na Espanha, engendram projetos articulados que buscam o fortalecimento e a ampliação dos sistemas universitários nos níveis local, nacional e mundial e, ao mesmo tempo, 
buscam provocar o fortalecimento dos campos político, econômico e cientifico hegemônico do mundo acadêmico. No Brasil, na Espanha e nas respectivas instituições acadêmicas, assim como em toda parte, observam-se cada vez mais interesses, tensões e estratégias visando à internacionalização da educação superior, da formação e produção do conhecimento de modo articulado ao processo de globalização da economia e interesses do capital.

A noção de uma sociedade ou economia do conhecimento tem circulado entre os objetivos, ações e estratégias para que novas pressões do campo político e econômico se interponham no espaço ou campo científico e universitário, alinhado à agenda global da educação. No caso brasileiro, as estratégias e ações de internacionalização têm sido voltadas mais fortemente ao incremento da cooperação internacional e ao fortalecimento do intercâmbio entre pesquisadores e docentes de outros países que mesmo se inserindo também na luta do campo político e científico atuam, a nosso ver, de forma mais tímida na busca do reconhecimento e prestigio local e internacional. A internacionalização tem sido uma constante nas políticas públicas nacional no Brasil, a exemplo das ações da Capes e do CNPq, bem como das metas e estratégias do PNE e nos PNPGs, enfocando a preocupação com a formação dos estudantes do país, o alargamento da pesquisa, da pós-graduação e da produção e difusão do conhecimento. A troca de experiência entre estudantes e pesquisadores de outros países certamente traz enriquecimento acadêmico pela via da inovação e enriquecimento cultural e científico, sem descurar dos interesses econômicos que também estão presentes.

O investimento do governo brasileiro para dar concretude a sua agenda de internacionalização tem privilegiado sobretudo as áreas das chamadas ciências duras. Muito embora reconheçamos a importância dessa priorização, observa-se também a necessidade que essa dinâmica se estenda igualmente para as demais áreas do conhecimento, para que esses processos de melhoria acadêmica tenham um movimento amplificado e no mesmo sentido. Nesse contexto, foi emblemático o caso do Programa Ciência sem Fronteiras que ganhou visibilidade no governo de Dilma Rousseff (2011-2016) como principal indutor deste processo de internacionalização. Embora não tenha sido objeto deste trabalho, vale registrar a existência de outras ações e iniciativas de internacionalização no Brasil via, sobretudo, a criação da Universidade Federal da Integração Latino-Americana (Unila) e a Universidade Integração Internacional da Lusofonia Afro-brasileiro (Unilab). Recentemente conforme abordado o Capes-Print tem se destacado como políticas de internacionalização das universidades brasileiras com ênfase na pós-graduação.

A Espanha, por sua vez, ao integrar o Espaço Europeu, tem sido privilegiada em estratégias de internacionalização de forma talvez mais sofisticada impondo aos sistemas nacionais de educação superior um nível de alinhamento horizontalizado, tendo como foco a qualidade e o desenvolvimento de competências e habilidades para enfrentar 
os desafios da sociedade atual - a sociedade do conhecimento - de modo a alcançar padrões de excelência mundial e de serem hegemonicamente visíveis. Ela deve servir de exemplo para outros países para que se formem uma real comunidade acadêmica uníssona em promover a melhoria de seus programas e de seus futuros profissionais.

Não se pode deixar em segundo plano, todavia, a compreensão de que a internacionalização da educação superior, em um mundo cada vez mais globalizado, ocorre num contexto de transnacionalização da educação superior, ou seja, de mercantilização e mercadorização em que agentes econômicos diversos dos países ricos estão cada vez mais interessados em ampliar os lucros com a oferta e expansão de serviços e produtos educacionais. Embora o campo científico tenha certa especificidade e autonomia, ele é cada vez mais pautado pelos interesses decorrentes do campo econômico, do campo político e do campo do poder e pela agenda globalmente estruturada. Assim, muitas das estratégias de internacionalização da educação superior ocorrem sem que se perceba o jogo que está sendo jogado no âmbito do poder global e que resulta em diferentes modos e processos de dominação dos países ricos em relação aos países pobres ou em desenvolvimento, que não ocupam lugar significativo na economia do conhecimento.

\section{Notas:}

1. Ver artículo de Beelen, J. (2011) "La internacionalización en casa en una perspectiva global: un estudio crítico del Informe del 3 Estudio Global de la AlU". En "Globalización de la educación superior" [monográfico en línea]. Revista de Universidad y Sociedad del Conocimiento, 2 (8), 85-100. UOC. http://www.redalyc.org/articulo.oa?id=78018793008

\section{Referências}

Azevedo, M. L. N. de \& Catani, A. M. (2015). "Educação Superior, Internacionalização e Circulação de ideias: ajustando os termos e desfazendo mitos". In Oliveira, J. F. de; Catani, A. M. (Orgs). Educação Superior e Produção do Conhecimento. Utilitarismo, Internacionalização e novo contrato social. Campinas, SP: Mercado das Letras.

Azevedo, M. L. N. de; Catani, A. M. \& Hey, A. P.(2017). "Circulação das ideias e internacionalização da Educação Superior: inferências a partir da teoria dos campos de Pierre Bourdieu", Revista Educação 3, (40), 296-304. http://revistaseletronicas.pucrs.br/ojs/index.php/faced/article/view/28980 Acesso: ago. 2019.

Brasil (2004). Coordenação de Aperfeiçoamento de Pessoal de Nível Superior (Capes). Plano Nacional de Pós-Graduação2005-2010. Brasília:Ministério da Educação.

Brasil (2010). Coordenação de Aperfeiçoamento de Pessoal de Nível Superior. Capes. Plano Nacional de Pós-Graduação 2011-2020. Brasília: Capes.

Brasil (2017). Coordenação de Aperfeiçoamento de Pessoal de Nível Superior. Capes. Print/Edital nº $41 / 2017$.

Bianchetti, L. \& Sguissardi, V. (2017). Da Universidade à Commoditycidade. Ou de como e Quando, se a Educação/Formação é Sacrificada no Altar do Mercado, o Futuro da Universidade se Situaria em Algum Lugar do Passado. Campinas: Mercado de Letras. 
Bourdieu, P. (1983).O campo científico. In: Ortiz, R. Pierre Bourdieu. Col. Grandes Cientistas Sociais. (pp.122-155). São Paulo: Ática.

Bourdieu, P. (2011). O campo político. Revista Brasileira de Ciência Política, 5, 193-216.

Bourdieu, P. (2006). O poder Simbólico. (6ª ed). Tradução Fernando Tomaz. Rio de Janeiro. Bertrand.

Bourke, A. (1997). The internationalisation of higher education - the case of medical education. Higher Education Quarterly, 51 (4), 325-46.

Conferência Mundial Sobre o Ensino Superior. Tendências de Educação Superior para o Século XXI. (1998) Paris: Unesco/Crub.

Conferência Mundial Sobre o Ensino Superior. Tendências de Educação Superior para o Século XXI. (2009) Paris: Unesco/Crub.

Craciun, D. (2018). National Policies for Higher Education Internazionalization: A Global Comparative Perspective. In Curaj, A., Deca, L. \& P, Remus. European Higher Education Area: The Impact of Pastand Future Policies. Cham (Switzerland): Springer Open.

European Union.(2018). The European Higher Education Area in 2018. Bologna Process Implementation Report. Brussels: Education, Audiovisual and Culture Executive Agency.

Lázaro, L. M. (2018).“Enseñar e investigar hoy en educación comparada: la perspectiva de tres décadas de dedicación académica en ese campo". Revista Brasileira de Educação Comparada, 1, (1), 1-7.

Marim, E. A. \& Brasil, W. (2004). Relatório Final Internacionalização da Educação Superior no Brasil (Convênio lesalc - Unamaz № 886446.3). Disponível em http://unesdoc.unesco.org/ images/0013/001399/139900por.pdf., consultado em julho de 2017.

Marrara, T. (2007). Internacionalização da Pós-Graduação: objetivos, formas e avaliação. Revista Brasileira de Pós-Graduação, 4, (8), 245-262.

Rede Eurydice. O Espaço Europeu de Ensino Superior em 2012. Relatório sobre a Implementação do Processo de Bolonha. Disponível em http://www.dgeec.mec.pt/np4/np4/\%7B\$clientServletPath\% 7D/?newsld=192\&fileName=O_Espaco_Europeu_de_Ensino_Superior_em_2.pdf, consultado em Julho de 2019.

Ruano-Borbalan, J.C. (2011). Globalização. In Van Zanten, A. (coord.) Dicionário de Educação. Petrópolis: Vozes.

Romão, J. E.(2008) Globalização e reforma educacional no Brasil (1985 - 2005). Revista Iberoamericana de Educación, 48, 111-127.

Santos, B. de S. (2002). Os processos da globalização. Eurozine. Disponível em http://www.eurozine. com/articles/2002- 08-22-santos-pt.html>, consultado março 2017

União Européia (UE) (2016). Estrategia para la Internacionalización de las Universidades Españolas 2015-2020. Marzo 2015.

Zgaga, P., Teichler, U., \& Brennan, J. (2013). The globalization challenge for European higher education: convergence and diversity, centers and peripheries. Frankfurt am Main: Peter Lang. 
Maria da Salete Barboza de Farias

Doutora em Sociologia, pós doutora e instância na Universidade de

Valência/Spain. Integra a Pesquisa - Mapa da Educação Superior na Paraíba - CNPq e o Grupo de Estudos em Educação Superior e Sociedade

Professora na Universidade Federal de Paraíba (Brasil)

E-mail: runasvida@gmail.com

ORCID: https://orcid.org/0000-0003-4302-3985.

João Ferreira de Oliveira

Doutor e Pós-doutor em Educação. Professor Titular da Faculdade de Educação da Universidade Federal de Goiás (UFG) e do Programa de Pós Graduação em Educação - (PPGE/UFG).

E-mail: joao.jferreira@gmail.com ORCID: https://orcid.org/0000-0002-4135-6340.

Luis Miguel Lázaro Lorente

Doutor em Filosofia e Ciências da Educação. Professor Catedrático da Universidade de Valência. Pesquisador visitante na Universidade da Califórnia, Los Angeles (UCLA) e Bangor (Reino Unido). E-mail: luis.lazaro@uv.es ORCID: https://orcid.org/0000-0003-3090-146X.

Correspondência:

Maria da Salete Barboza de Farias Universidade Federal da Paraíba - Campus I

Centro de Educação (CE) Programa de Pós-Graduação em Políticas Públicas, Gestão e Avaliação da Educação Superior (MPPGAV) Cidade Universitária - CEP 58051-900 - João Pessoa-PB-Brasil

Data de submissão: Setembro de 2019

Data de avaliação: Dezembro de 2019

Data de publicação: Novembro 2020 